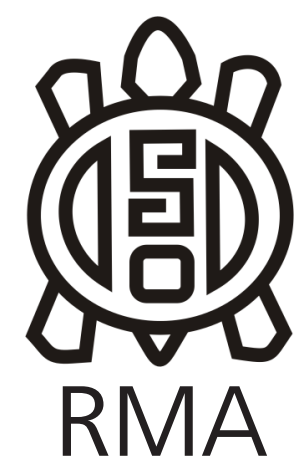

Dossier

\title{
La producción de grandes soportes alargados en las ocupaciones antiguas de Serranópolis, Brasil Central
}

\author{
The production of large elongated blanks in the ancient occupations of \\ Serranópolis, Central Brazil
}

\author{
Maria Jacqueline Rodet*, Déborah Duarte-Talim**, Jacques Pelegrin*** \\ y Pedro Ignácio Schmitz****
}

*Faculdade de Filosofia e Ciências Humanas, Departamento de Antropologia e Arqueologia, Universidade Federal de Minas Gerais. Museu de História Natural e Jardim Botânico-UFMG, Belo Horizonte, Minas Gerais, Brasil. Laboratoire Préhistoire et Technologie (UMR7055), Université Paris-Ouest Nanterre la Défense, Paris, Francia. E-mail: mjrodet.ufmg@gmail.com **Museu de História Natural e Jardim Botânico-UFMG, Belo Horizonte, Minas Gerais, Brasil. E-mail: delsduarte@hotmail.com ***Centre National de la Recherche Scientifique (CNRS), Laboratoire Préhistoire et Technologie (UMR7055), Université Paris-Ouest Nanterre la Défense, Paris, Francia. E-mail: jacques.pelegrin@mae.u-paris10.fr ****Instituto Anchietano de Pesquisas, Universidade do Vale do Rio dos Sinos, São Leopoldo, Rio Grande do Sul, Brasil. E-mail: anchietano@unisinos.br

\begin{abstract}
Resumen
La región de Serranópolis, (Goiás, Brasil) se inserta en el área definida como Brasil Central. Los estudios de las industrias líticas de los niveles antiguos del yacimiento arqueológico GO-JA-03, fechados en $9.765 \pm 75$ AP, indicaron una producción de grandes lascas alargadas (máximo $20 \mathrm{~cm}$ ), producidas en una arenisca local altamente silicificada. Las lascas sirvieron como soporte para la producción de instrumentos unifaciales muy poco espesos y también de instrumentos unifaciales de sección plano-convexa, más gruesos. El objetivo de este trabajo es realizar un análisis tecnológico basado en la conceptualización de cadenas operativas y enfocado a las etapas de producción de los instrumentos, con énfasis en las técnicas utilizadas durante las fases iniciales, cuya finalidad es la obtención de los soportes. Los resultados del análisis tecnológico son comparados con un programa experimental y apuntan a la utilización de dos técnicas principales: la percusión directa orgánica y la percusión tangencial de piedra suave - poco comunes en la etapa de débitage en las industrias líticas brasileñas. La contrastación de la utilización de estas técnicas en esta etapa dela talla es coherente con el alto grado de savoir-faire observado en las industrias líticas de las ocupaciones antiguas del sitio.
\end{abstract}

Palabras clave: Percusión orgánica; Percusión tangencial con piedra dura; Tecnologia lítica; Serranópolis; Instrumentos unifaciales.

\begin{abstract}
The region of Serranópolis (Goiás, Brazil) is inserted in the area defined as Central Brazil. The studies of the lithic industries of the ancient levels of the archaeological site GO-JA-03, dated at $9765 \pm 75$ BP, indicate a production of large elongated flakes (the longer are $20 \mathrm{~cm}$ ), produced in a highly silicified local sandstone. The flakes were used as blanks to produce very thin unifacial tools and other thicker flat-convex section unifacial tools. The objective of this work is to realize a technological analysis based on the concept of Chaïne Opératoire and focoused on the tools' production phases, emphasizing the technological stigmas that indicate the techniques used during the initial phases, in order to obtain thes blanks. The results of the technological analysis are compared to an experimental program and point out the use of two main techniques: organic direct percussion and soft stone tangential percussion - uncommon in the débitage stage in the lithic Brazilian industries. The verification of the use of these techniques in this phase of knapping is consistent with the high degree of savoir-faire observed in the lithic industries of the ancient occupations of the site.
\end{abstract}

Keywords: Organic percussion; Tangential percussion with hard stone; Lithic technology; Serranópolis; Unifacial tool.

La región de Serranópolis, en el sudoeste del estado de Goiás (Brasil), es un área con importantes yacimientos arqueológicos y se inserta en la región definida como
Brasil Central, con aproximadamente 2 millones de $\mathrm{km}^{2}$, compuesta por los estados de Minas Gerais, Bahia, Pernambuco, Rio de Janeiro, Espírito Santo, Goiás y Tocantins 


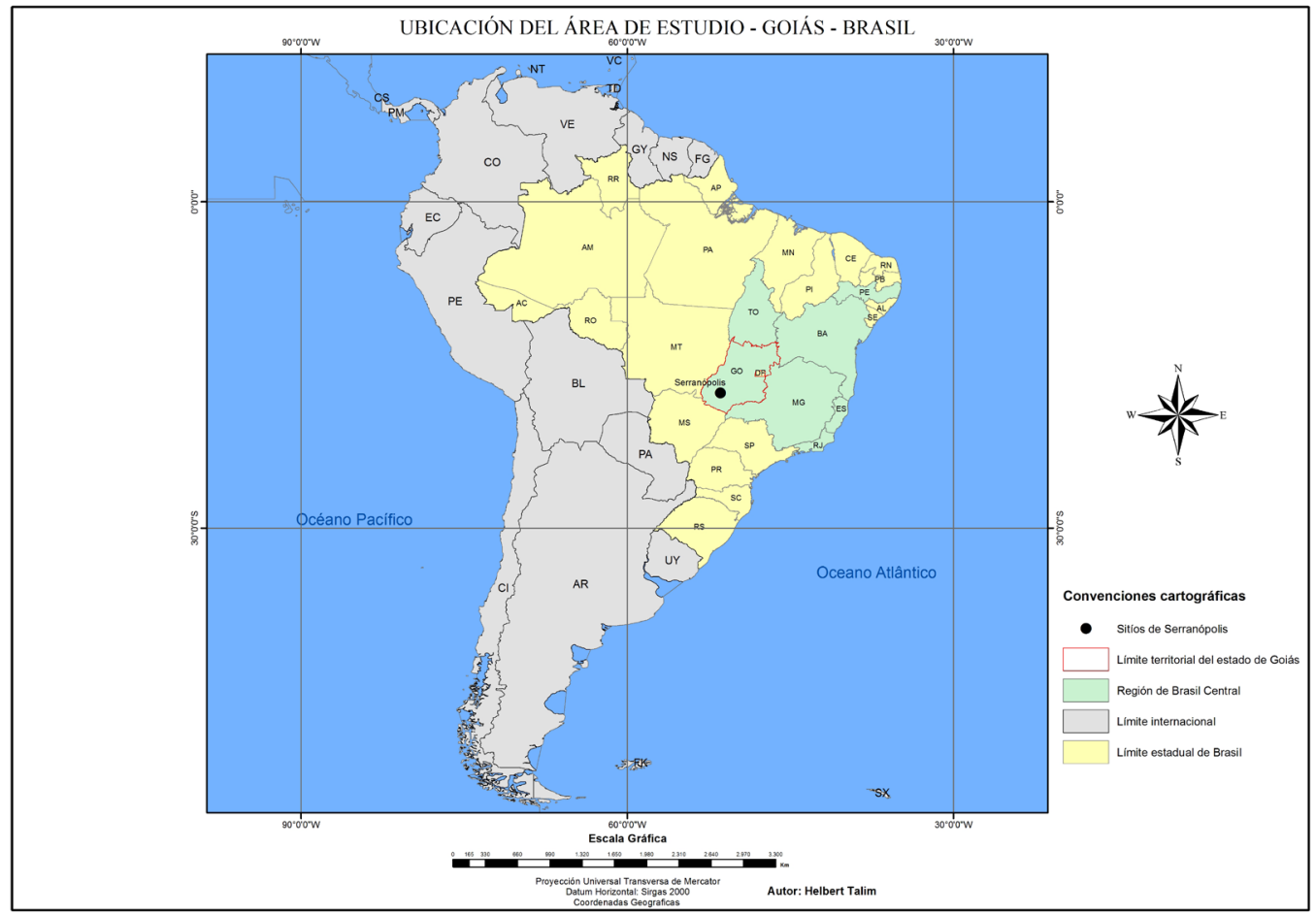

Figura 1. Mapa de localización del área de estudio, en el contexto de Brasil Central (Schmitz et al. 2004, modificado por H. Talim).

Figure 1. Map of the location of the study area, inserted in the context of Central Brazil (Schmitz et al. 2004, modified by H. Talim).

(Figura 1). La región de Serranópolis presenta un bioma típicamente de Cerrado, sustentado por un substrato rocoso de arenisca (ArenitoBotucatu) que, localmente, tuvo contacto con las lavas basálticas de la Formação Serra Geral, generando una materia prima silicificada, de alta calidad para la talla. Esta investigación presenta las industrias líticas realizadas sobre esta materia prima, en los niveles más antiguos de dos de los sitios arqueológicos, desde hace más de 10 mil años $(9.765 \pm 75$ AP, SI-3110 - cal 11.261 hasta 10.784 AP. calibración OxCal, 2 sigma ${ }^{1}$, para la ocupación de GO-JA-03).

Las investigaciones sistemáticas en la región de Serranópolis empezaron en el año de 1975, y, bajo la dirección del P. I. Schmitz. Como resultado, se encontraron 40 yacimientos arqueológicos a la base de afloramientos rocosos (abrigos), en una altitud entre $950 \mathrm{~m}$ y $500 \mathrm{~m}$. Las fechas más antiguas ubican las ocupaciones en el límite entre los periodos Pleistoceno y Holoceno, siendo las más antiguas de 10.580 $\pm 1150 \mathrm{AP}$ (SI-3699, 12.744-11.834 años cal. AP) y las más recientes de $925 \pm 60$ BP (SI-3690, 956-667 años cal. AP), en la ocupación de GO-JA-01 (Schmitz, Rosa y Bitencourt, 2004). Las excavaciones fueron realizadas por niveles artificiales de $10 \mathrm{~cm}$ y el sedimento, proveniente de la descomposición de la roca

\footnotetext{
${ }^{1}$ La calibración de las fechas presentes en este artículo fue hecha a través del programa online OxCal V.4.3.2 (Ramsey, 2009), basado en la curva SHCal-13 (Hogg et al., 2013), adoptando un nivel de probabilidad de $95 \%$ (2 sigma).
}

de caja, está compuesto por una arena muy fina y difícil de mantener in situ durante las excavaciones, además de materiales antropogénicos. Los vestigios exhumados en las diversas excavaciones pueden ser sintetizados como una gran cantidad de restos líticos y materiales orgánicos (e.g., tales como maíz, algodón, restos de frutas, cuerdas, etc.). Las paredes del abrigo están cubiertas por pinturas y grabados (Schmitz et al., 2004).

Los yacimientos arqueológicos tomados como referencia son GO-JA-03, donde se recuperaron cerca de 81.624 piezas, instrumentos, núcleos y desechos restos de talla (débris de débitage). También se incluyeron dos piezas muy elaboradas de la ocupación de GO-JA-14, las cuales complementan las intenciones de débitage observadas en el primer sitio. Los dos yacimientos arqueológicos están cercanos entre sí, aproximadamente $6 \mathrm{~km}$, y presentan una estratigrafía muy parecida. En la pequeña base elevada del sitio GO-JA-03 (Figura 2) hay una gran concentración de materiales tallados: parece que los talladores se sentaban allí para tallar. La gran cantidad de material y las particularidades de la muestra del pequeño sector excavado $(2,5 \times 2,5 \mathrm{~m}$ y $2,40 \mathrm{~m}$ de profundidad), indican una utilización constante del sitio a lo largo de los años, como un lugar de producción de instrumentos y de soportes. Hay muchos restos de talla (débitage) y pocos instrumentos. Los artefactos que fueron dejados no son los mejores representantes de los 
Planta baixa do sítio GO-JA-03
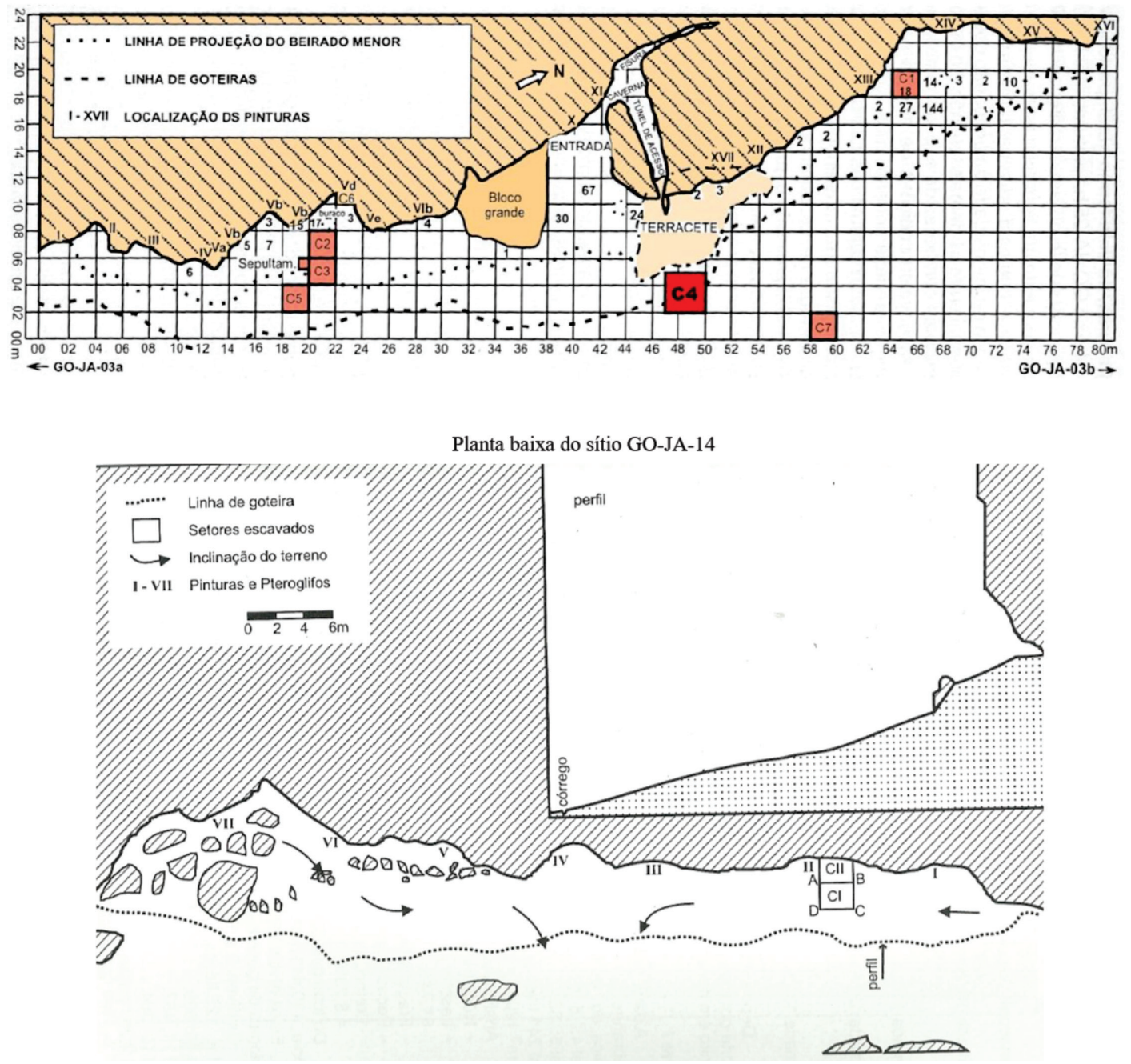

Figura 2. Vista en planta de los yacimientos arqueológicos presentados. Arriba, planta del yacimiento GO-JA-03, con el Corte IV destacado (Schmitz et al. 2004, modificado por J. C. Moreno de Sousa, 2014: 63) y, abajo, planta del sitio GO-JA-14, alrededor de 6 km (Schmitz et al. 2004: 153).

Figure 2. Ground floor plans of the archaeological sites presented. Above, GO-JA-03 site plan, with Cut IV highlighted (Schmitz et al. 2004, modified by J. C. Moreno de Sousa, 2014: 63) and, below, GO-JA-14 site plan, distant approximately 6 km (Schmitz et al. 2004: 153).

productos finales buscados. Sin embargo, al desarrollar el análisis tecnológico en una localidad de producción lítica, se observan diferentes etapas de distintas cadenas operativas mezcladas. El objetivo final, los instrumentos, normalmente no se registran en el yacimiento de producción, siendo posiblemente llevados fuera de la localidad. Solamente los instrumentos que presentan fallas, aquellos que se fragmentaron durante el proceso de producción, o los instrumentos agotados (exausted tools), al final de su vida útil, fueran descartados en el lugar (Rodet, Duarte-Talim y Schmtiz, 2019). Según J. Pelegrin (2005), se puede esperar que algunos pocos instrumentos terminados pudiesen ser olvidados en la localidad, pero la cantidad no puede sobrepasar $6 \%$ de la colección. Por otro lado, no se puede olvidar a los aprendices. Ciertamente, alrededor de los talladores experimentados había niños, en distintos niveles de aprendizaje. De acuerdo con C. Chauchat (1991), en este contexto se pueden observar las piezas desviadas de la ejecución óptima. Estas piezas guardan, en parte, las normas establecidas por el grupo, pero presentan errores de producción, por ejemplo, muchos accidentes o producciones en materias primas de menor calidad (Rodet et al., 2019).

En este marco, el objetivo de esta investigación es presentar las etapas de producción de los soportes del grupo de los instrumentos unifaciales largos, más y menos espesos. Se pretende dar énfasis en los estigmas tecnológicos que indican las técnicas utilizadas durante 
las fases iniciales de la producción. Uno de los propósitos de este articulo es demonstrar que la intención de la producción presente en Serranópolis está muy alejada de la homogeneidad propuesta para el Brasil Central (ver más adelante) y demostrar que se trata de la producción de soportes alargados por percusión directa de piedra blanda (golpeado en el interior del núcleo) y/o por percusión orgánica. Al mismo tiempo, las observaciones arqueológicas fueron sostenidas por un programa experimental realizado sobre una arenisca silicificada, pero no la misma roca de Serranópolis. Consideramos relevante discutir ciertos aspectos de las industrias líticas de aqquellas poblaciones que se instalaron en Brasil Central aproximadamente hace 12.000 y 10.000 años AP. Estas industrias fueron percibidas durante muchos años como homogéneas desde el punto de vista tecnológico, dado que los instrumentos unifaciales fueron muchas veces vistos como poco elaborados y sus soportes siempre obtenidos por percusión directa dura.

\section{Las industrias antiguas del Brasil Central: discusión general}

Las industrias líticas correspondientes a las poblaciones antiguas que se instalaron en el Brasil Central entre aproximadamente los 12.000 y 10.000 años AP (no calibrados) fueron percibidas como homogéneas desde el punto de vista tecnológico, durante muchos años. Frecuentemente denominadas "Tradição Itaparica" y actualmente puesto en debate por algunos autores (Bueno, 2007; Lourdeau, 2010; Rodet, 2006; Rodet, Duarte-Talim y Bassi, 2011; etc.), ese término fue inicialmente sostenido en los años 1960 por V. Calderón (1969), a partir de industrias encontradas en la orilla del río São Francisco, en el estado de Pernambuco, en la Gruta do Padre, durante la implantación del Programa Nacional de Pesquisas Arqueológicas (PRONAPA). Más tarde, el término fue apropiado y reutilizado por los arqueólogos del resto del Brasil para referirse a las industrias antiguas y con un buen control de la talla (Rodet et al., 2011), aquellas relacionadas a la transición entre los periodos Pleistoceno/ Holoceno y Holoceno Inicial (aproximadamente entre 12.000 y $10.000 / 9.000$ años AP).

Sin embargo, esta definición de facto presenta elementos limitantes para una utilización genérica. El primero de ellos es que la definición fue basada en análisis tipológicos y no tecnológicos realizados sobre "fósiles guías", constituidos por instrumentos unifaciales sobre lascas de cantos rodados, tales como el denominado "plano-convexo". El segundo problema es que las dataciones para los niveles más profundos de las industrias excavadas en la Gruta do Padre tienen un rango cronológico que va de los $7.580 \pm$ 410 AP (Si-644, 9.941-7.308 años cal AP) a los $2.360 \pm 50$ AP (CSIC805, 2.703-2.150 años cal AP - Calderón, 1969; Martin, 2013). Esto indicaría que se trata de poblaciones relacionadas a los periodos del Holoceno medio y reciente, y no poblaciones antiguas de la transición entre los periodos Pleistoceno y Holoceno.

Por otro lado, estudios actuales realizados en diversas áreas del Brasil Central (Bassi y Rodet, 2011; Boëda et al., 2014; Bueno, 2007; Isnardis, 2009; Lourdeau, 2010; Moreno de Sousa, 2014; Martins y Kashimoto, 2012; Rodet, 2006; Rodet et al., 2011; Vieira et al., 2015; entre otros) demuestran una variedad de elecciones de las poblaciones antiguas para la producción de sus instrumentos unifaciales, más que una conformidad de industrias líticas. Diferentes materias primas, soportes, técnicas y métodos fueron seleccionadas en la producción de esas diversas industrias líticas. Aunque la intención sea producir instrumentos a partir de lascas planoconvexas, las formas de realizarlas son distintas. Por ejemplo, en la región del alto medio río São Francisco (municípios de Buritizeiro, Jequitaí, Lagoa dos Patos, Januária, Diamantina, etc.), se observan sistemáticamente distintas intenciones y elecciones tecnológicas para el periodo en referencia. Mientras que en la región de Buritizeiro, orilla del rio São Francisco, los grandes cantos rodados de cuarcita tuvieron un rol importante en las producciones de instrumentos unifaciales y de núcleos de fatiagem ${ }^{2}$ de lascas. Más al norte, en la región de Januária e Itacarambi, valle del río Peruaçu, el silexito y la arenisca fueron las materias primas preferenciales para producir instrumentos unifaciales y bifaciales elaborados, y también una industria simple, poco transformada, sobre lascas y fragmentos (Prous, Fogaça y Alonso, 1994; Rodet 2006; Rodet et al., 2011; entre otros).

En Diamantina, la base de la producción de instrumentos unifaciales fue una cuarticita muy fina, de excelente calidad para latalla (Formação Galho do Miguel). Además, el cuarzo hialino que sirvió para la producción de piezas bifaciales delgadas y instrumentos unifaciales, a partir de un "lascamento refinado", que incluye la utilización de la percusión blanda (Isnardis, 2009). Estos ejemplos deben ser tomados con cuidado, pues otras dimensiones importantes deben entrar en un análisis regional, como las dataciones sistemáticas, las ubicaciones de los yacimientos arqueológicos en relación con el relieve y el tipo de substrato. Deben ser considerados los distintos tipos de ocupaciones (por ejemplo, producción, consumo, caza, etc.), pues cada uno de ellos tendrá conjuntos de vestigios líticos específicos, además de las ubicaciones de los yacimientos en relación con los grandes y pequeños ríos. Finalmente, no se puede olvidar la representatividad de las ocupaciones prehistóricas que están, principalmente, basadas en un solo tipo de sitio: aquellos bajo abrigos rocosos. Ese tipo de yacimiento representa solamente una

\footnotetext{
${ }^{2}$ Fatiagem es un término utilizado para referirse a los diferentes métodos de débitage de los cantos rodados. Los talladores hacen la gestión racional de la extracciónde lascas sobre esos soportes, tornándo los muy rentables. A pesar de presentar una fórmula simple de débitage, los talladores invierten un tiempo mayor en laelección de los soportes nódulos, anticipándose, así, el método a ser utilizado y las lascas buscadas (Inizan; Reduron-Ballinger, Roche y Tixier, 2017)
} 
de las dimensiones de la vida de los grupos humanos del pasado y no puede ser tomado sin una reflexión crítica, como un retrato fidedigno de esas poblaciones.

En general, las otras áreas de la gran región denominada Brasil Central son similares los casos anteriores, es decir, la variabilidad de las industrias líticas antiguas (transición Pleistoceno/Holoceno) es confirmada por los datos provenientes de los estados de Tocantins, de Mato Grosso do Sul, de Goiás y de Bahia. Por ejemplo, en el estado de Tocantins, en la región del Lajeado, se destaca la presencia de sitios a cielo abierto con fechas del Holoceno inicial y una industria lítica compuesta por: i. producción de instrumentos más elaborados sobre lascas de sección plano-convexa, con gran variabilidad morfológica, sobre un arenisca silicificada de granulometría muy fina y de origen exógeno; ii. junto a una industria más simple, elaborada sobre cantos rodados de cuarcita y cuarzo, materias disponibles en los alrededores de los yacimientos y iii. una discreta producción de instrumentos bifaciales, delgados o no, en arenisca silicificada, filito y cuarzo (Bueno, 2007). Finalente, en el nordeste del estado de Mato Grosso do Sul, en la cuenca del río Paraná, el registo de la transición del Pleistoceno/Holoceno y del Holoceno inicial, muestra la utilización de cantos rodados de arenisca silicificada, silexito y bloques de cuarticita para la producción de instrumentos unifaciales de sección planoconvexa, más o menos espesos, o incluso achatados, sobre lascas (Martins y Kashimoto, 2012).

Entonces, los antecedentes generan y apoyan la dificultad de considerar el periodo antiguo de las ocupaciones en el Brasil Central como algo homogéneo. Sumado a ello, es muy probable que distintos grupos ocuparan este espacio, cada uno con industrias líticas particulares. Separarlas en el tiempo y en el espacio y comprender sus dinámicas, es un trabajo arduo y requiere de muchos años y equipos de estudio en la región, además de análisis comparativos de las distintas colecciones.

Siguiendo este abordaje tecno-económico, la cuestión de la heterogeneidad de las industrias de este periodo aparece cada vez más clara en los estudios publicados. No obstante, existen puntos comunes que sobresalen: la búsqueda de instrumentos unifaciales de sección planoconvexa, sobre lascas más largas que anchas, la obtención de productos alargados pero achatados, la producción de soportes con una gran cantidad de bordes cortantes disponibles, la frecuente presencia de instrumentos que indican un alto nivel de savoir-faire y la elección de materias primas específicas y de buena calidad para la producción de instrumentos más elaborados. Estas intencionalidades, han sido sistemáticamente observadas en conjunto con una industria más simple, no solamente en el Brasil Central, pero también en un sector específico de la Amazonia brasileña, como indican los estudios recientes de las industrias de los niveles más antiguos de la Caverna da Pedra Pintada, estado del Pará (Rodet,
Duarte-Talim y Pereira, 2017; Roosevelt et al., 1996). A estos conjuntos podemos añadir la producción bifacial.

\section{Marco Teórico-Metodológico}

El método de análisis se basa en los conceptos de Cadena Operativa y Análisis Tecnológico, así como también en un programa experimental realizado en conjunto con el Laboratoire de Préhistoire et Technologie (Prétec) de Paris Oeste. El concepto de cadena operativa, adaptado de la Escuela Francesa (Inizan et al., 2017; Leroi-Gourhan, 1964; Mauss, 1947; Tixier, 1978; etc.), es fundamental en este estudio pues permite volver a trazar conjuntos de acciones antrópicas realizadas sobre el material bruto, pasando por la elección de la materia prima, las diferentes fases de producción y el abandono del misma. Esta noción general permite reconstituir los comportamientos en una perspectiva paleoetnológica, haciéndose así, un instrumento de análisis. La clasificación según las fases de las cadenas operativas es jerarquizada y tiene la intención de evidenciar una organización diferenciada en el espacio y en el tiempo, es decir, qué etapas fueron realizadas dentro del yacimiento arqueológico y lo que puede haber sido llevado por los grupos. Conectada a ese concepto, la lectura tecnológica de los distintos tipos de vestigios permite entender la intención de la producción. Se trata de comprender cómo los brutos de débitage, façonnage y retouche se vinculan y de qué manera las diversas fases de producción de los objetos se organizan en el tiempo y en el espacio.

Los artefactos son imágenes mentales (más o menos completas) a los que el tallador recurre, en la ausencia del objeto mismo (Pelegrin, 1991). Los grupos van en búsqueda de sus memorias sociales para la producción de industrias líticas, dentro de una gama de posibilidades, gestos y técnicas. Se trata de elecciones culturales de alguien que aprendió para enseñar en continuidad. Según J. Pelegrin (1991), es esencial que el arqueólogo pueda identificar las maneras de percibir de una determinada sociedad (conceptos de forma o morfología) y las maneras de hacer (modalidades preferenciales) presentes en la colección a ser estudiada. Entre esas maneras de ver y de hacer, el conocimiento - y la utilización - de las técnicas de lascamiento es un capítulo importante, pues la práctica de una técnica está, seguramente, muy enraizada dentro de la tradición técnica de un grupo o de una lignée humana.

La técnica (o eventualmente las técnicas) de débitage de grandes soportes sobre los cuales fueron confeccionados las piezas plano-convexas estudiadas, merecen así una precisión. Pero, el reconocimiento de las técnicas de lascamiento es un trabajo delicado, pues ellas son como fantasmas: podemos describir los objetos de piedra tallados, pero no vemos directamente sobre ellos, a partir de cual (o cuales) técnica (s) con que fueron producidos. Para eso, debemos hacer un "desvío" y pasar por un referencial experimental. Es comparando de cerca los 
estigmas y las tendencias morfológicas obtenidas por la reproducción experimental de una o otra técnica, que podemos reconocer esta misma técnica sobre el material arqueológico (o, por lo menos, sostener una discusión comparativa entre las señales de una o otra técnica, o sea, un diagnóstico - como en medicina).

Con la intención de constituir un referencial adaptado a la materia prima en cuestión (arenisca silicificada, brillante, que recibió un tratamiento térmico natural por el contacto local de una lava de basalto), fue elegido un gran bloque de arenisca bien silicificado, de apariencia brillante. En un primer momento, se realizaron una serie de extracciones alargadas por percusión directa con piedra blanda (el percutor fue un canto rodado de arenisca incompletamente silicificado, generando un contacto blando). Seguidamente, se realizaron una serie de extracciones de lascas alargadas a partir de un percutor orgánico (madera dura, buis, aproximadamente $800 \mathrm{~g}$ ). De esta manera se observa que mediante la aplicación de estas dos técnicas pueden obtenerse lascas alargadas, regulares y con tendencia espesa, aptas a ser transformadas en instrumentos plano-convexos. Es preciso, entonces, concentrarse en los estigmas técnicos, los cuales son resultados de la interacción entre el plan de percusión y el percutor, y definidos por la forma y reacción al impacto.

Durante la experimentación, el percutor de piedra blanda fue utilizado golpeando cerca del borde, después de la extracción de la cornisa, en el interior de la plataforma de percusión (aproximadamente 1 a 1,5 cm cerca del borde). La plataforma es de morfología lisa y forma un ángulo de aproximadamente $80^{\circ}$ con la superficie de débitage (condiciones próximas el ideal para esta técnica). En cada uno de los casos, el impacto aparece bien visible, bajo la forma de una fisura al menos parcial, que completa el desvío que forma el punto de impacto, de aproximadamente 5 mm de diámetro (Figura 3), en el nivel de la línea posterior (entre el talón y la cara inferior de la lasca).

Las extracciones con el percutor orgánico, luego de la abrasión y emoussé del sector del futuro talón en la plataforma de percusión, presentan un aspecto bien distinto (Figura 3): no hay fisura, ni punto de impacto. En este sentido, el percutor de madera entra en contacto con una superficie relativamente importante del borde de la plataforma de percusión (más de $1 \mathrm{~cm}$ de ancho 3 a 6 $\mathrm{mm}$ de espesor) y desprende esta superficie, rasgándola. Este efecto se traduce en la lasca en la forma de un labio ${ }^{3}$ muy claro que aparece detrás del talón.

\section{Las técnicas y intenciones de la producción}

En el sitio GO-JA-03, la industria es producida en una

\footnotetext{
${ }^{3}$ Labio es un pequeño desdoblamiento regular a lo largo de toda la
} línea posterior arenisca local altamente silicificada (98,84\%, 80.415 piezas). Las otras materias primas son anecdóticas en la colección (basaltos, 7 piezas, 0,08\%; calcedonia, 938 piezas, 1,15\%; otras materias líticas, 14 piezas, 0,001\%), lo que indica que los talladores buscaban exactamente esta materia prima, que es de alta calidad para la talla.

A pesar de que es un amas de débitage (acumulación de restos de talla), fue posible entender algunas de las intenciones de producción. Las lascas buscadas como soporte son grandes, anchas (hasta $20 \mathrm{~cm}$ de ancho y muchas otras tienen alrededor de $15 \mathrm{~cm}$ ) y espesas a muy delgadas. Fueron observadas cadenas operativas de producción de instrumentos unifaciales y bifaciales. En este trabajo se presentarán los dos principales resultados relacionados a los instrumentos unifaciales, realizados sobre soportes distintos: i. soportes alargados, generalmente anchos y espesos y ii. soportes alargados, menos anchos y muy poco espesos.

La producción de lascas grandes, anchas, largas, normatizadas y con un determinado espesor que serán transformadas en instrumentos unifaciales de sección plano-convexa, es realizada por percusión directa tangencial de piedra blanda, o por percusión directa dura. Los soportes frecuentemente tienen talones pequeños ${ }^{4}$ (en relación con las dimensiones de la lasca), en general lisos, el sector proximal poco espeso, en contraste con la parte distal más espesa. Las caras inferiores son planas, sin bulbos marcados y raramente presentan con raros accidentes de talla. Tres procedimientos técnicos específicos demuestran una intención en preparar muy bien los talones, evidenciándoles y, al mismo tiempo, volviéndolos resistentes. Los procedimientos de preparación del talón incluyen: abrasión intensa, emoussé y pequeñas extraccíones en el entorno de la futura zona del punto de impacto. La abrasión intensa permite entrar en la masa del núcleo y genera lascas con el sector proximal poco espeso. Juntos, abrasión y emoussé, crean un pequeño sector liso, sin asperezas y resistente, perfecto para recibir el golpe del percutor y permitir la iniciación de la fractura concoidal. Un pequeño talón, resistente, permite la concentración de energía y la iniciación de la fractura. Al contrario, talones grandes dispersan la energía. En una materia prima de buena calidad para la talla (homogénea, granulometría fina, libre de fisura, incrustaciones, etc.), un buen percutor y un gesto preciso, hacen que la fractura concoidal se desarrolle fácilmente hasta la distancia deseada, sin accidentes.

Solamente los percutores blandos orgánicos pesados, o de piedra blanda, combinados con gestos específicos tangenciales, y con el plano de percusión muy bien preparado para soportar la presión del golpe, son capaces de iniciar la fractura a partir de talones tan pequeños, sin

\footnotetext{
${ }^{4}$ Dimensiones de los talones: $2,1 \times 1 \mathrm{~cm} ; 0,6 \times 0,4 \mathrm{~cm} ; 2,7 \times 1,1 \mathrm{~cm}$ y $4,2 \times 2 \mathrm{~cm}$, en lascas sistemáticamente más anchas que $15 \mathrm{~cm}$.
} 
A

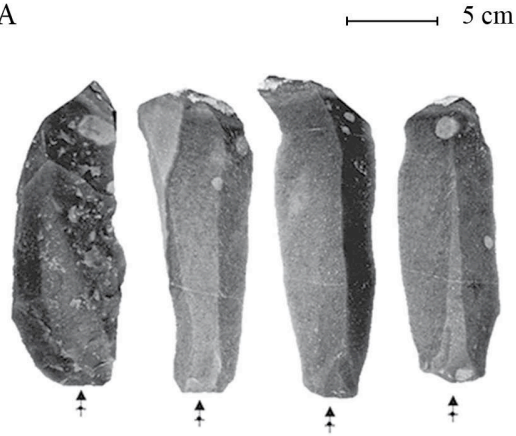

B

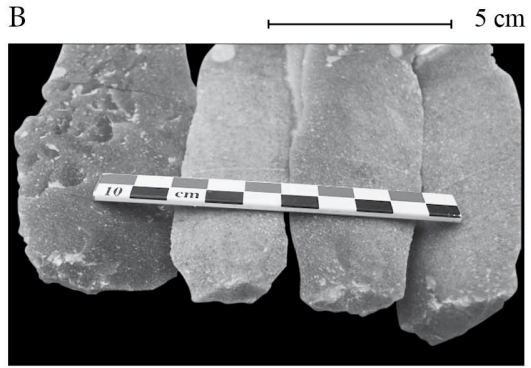

C

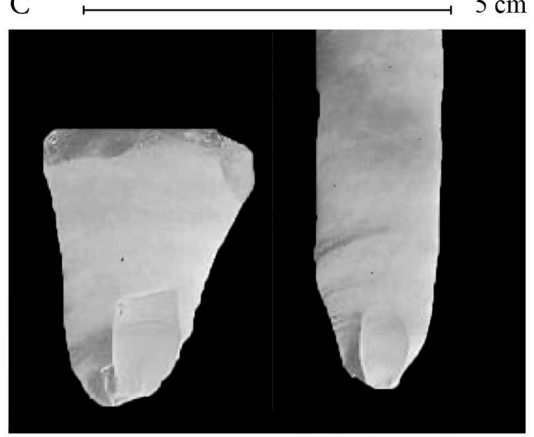

$\mathrm{D}$

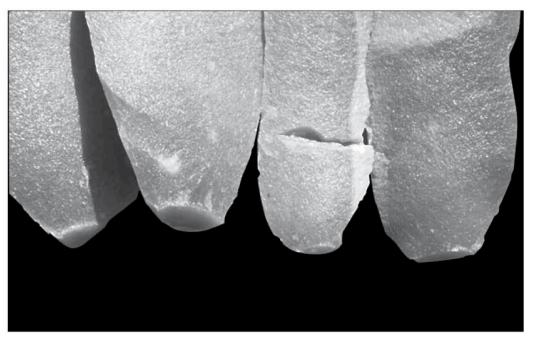

Figura 3. Test experimental de J. Pelegrin. A: cuatro lascas alargadas retiradas por percutor de piedra blanda (cara superior); B: cara inferior, mostrando los talones y la fisura que forma el punto de impacto; C: ejemplos de lascas con esquirlamiento del bulbo (experimental); D: cuatro lascas alargadas, retiradas por percusión orgánica (madera), cara inferior.

Figure 3. J. Pelegrin's experimental test. A: four elongated flakes removed by soft stone hammer; $\boldsymbol{B}$ : lower face, showing the butts and the fissure that forms the impact point; $\boldsymbol{C}$ : examples of flakes with bulb scars (experimental); $\boldsymbol{D}$ : four elongated flakes, removed by organic percussion (whith wood), lower face. fragmentar el borde del núcleo. Ellos absorben parte del golpe y crean lascas largas, regulares, planas en las caras inferiores y libres de bulbos marcados.

\section{Discusión}

La observación de preparaciones sistemáticas y minuciosas en el material arqueológico, así como en el pequeño programa experimental, corroboran nuestro diagnóstico. En complemento, se registra sobre el material arqueológico un accidente denominado esquirlado del bulbo. Según J. Pelegrin (2000), se trata de un accidente relacionado frecuentemente a la percusión tangencial de piedra blanda. Sin embargo, no podemos olvidar que cuanto mayor es el producto extraído, mayor es la fuerza utilizada, y, en contraparte, es posible un mayor número de accidentes. Todavía, mismo se la arenisca es muy silicificada se trata de una materia prima porosa, más porosa que el sílex o el silexito.

En las caras superiores, los negativos presentes indican el predominio de núcleos con una plataforma de percusión preferencial: la mayoría de los negativos están en el mismo sentido de la lasca o con un leve desplazamiento del eje de percusión. Sin embargo, no se puede olvidar que, por ser un área de taller, los productos de primera elección, aquellos que serán transformados en instrumentos, no se encuentran en el lugar, fueron llevados.

El inicio del débitage se realizó ciertamente sobre los grandes bloques de arenisca silicificada, como indican las grandes lascas primarias con restos de corteza o subcorticales espesas, con intrusiones y negativos en distintas direcciones. Se trata de núcleos que pueden llegar hasta $70 \mathrm{~cm}$ o $50 \mathrm{~cm}$ de diámetro, de los cuales fueran extraídas lascas de dimensiones próximas a $30 \mathrm{~cm}$ de largo (o más), $25 \mathrm{~cm}$ de ancho y $10 \mathrm{~cm}$ de espesor (o más). La exploración de los bloques, que serían transformados en núcleos, fue realizada i. directamente por percusión sobre las paredes de los abrigos ocupados (como es el caso del sitio GOJA-01 - Figura 4), en uno de los estratos específico de la roca, o ii. sobre las torres residuales de materia prima de excelente calidad para la talla que existen en las proximidades inmediatas de los abrigos. El fuego también puede haber sido uno de los mecanismos de extracción de los bloques, como indican algunas grandes lascas del inicio del débitage que muestran escamas y cúpulas térmicas en las caras inferiores y superiores.

El tratamiento inicial de los núcleos, observado a partir de las grandes lascas de inicio de débitage, demuestran la intención de buscar una superficie adecuada para la extracción de las lascas grandes y largas, con determinado espesor. Los accidentes observados, aunque no son muy frecuentes, incluyen fracturas siret, el esquirlamiento del bulbo y mas escasas, o réfléchissement (el reflejado). Varios productos de inicio de débitage son testimonios de una puesta a punto (mise en forme) de adelgazamiento y calibración del núcleo: lascas espesas corticales y subcorticales, con presencia de negativos indicando distintos planos de percusión, talones lisos o corticales, largos, a veces preparados (abrasión o pequeñas extracciones alrededor), otras por la presencia de bulbos marcados, retiradas por percusión directa con piedra dura o piedra blanda, etc. Algunas lascas muestran claramente la intención de limpiar la superficie de débitage ya que se trazan en sus caras superiores sectores reflejados (réfléchissements) o con aristas. La materia prima de estos productos es, algunas veces, más porosa, indicando 


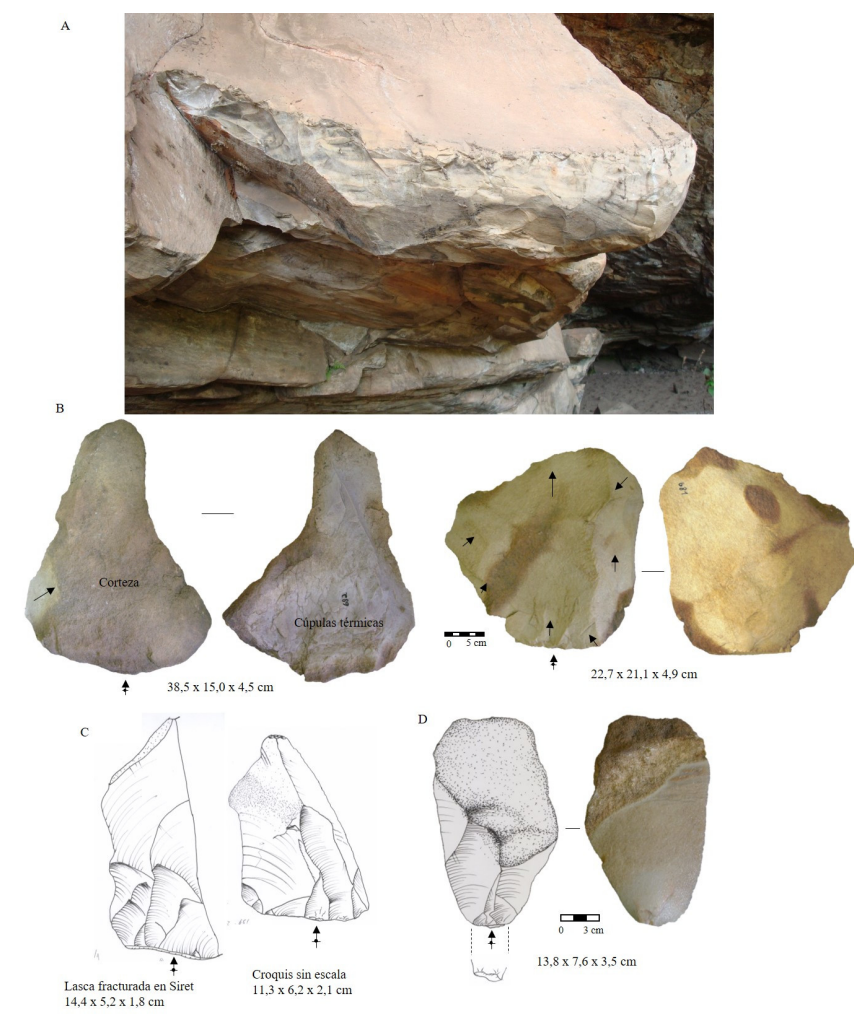

Figura 4. Núcleo y lascas de débitage. A: paredón rocoso dentro del abrigo, con negativos de extracciones (Foto: A. da Costa); B: grandes lascas de débitage, anchas y compactas, retiradas por percusión directa dura, con estigmas de contacto térmico, además de corteza. También son relacionadas con la limpieza y preparación de las superficies del débitage; C: Lascas iniciales de débitage, más alargadas que anchas, con talones también largos y espesos, accidente Siret y corteza. D: Lasca inicial de débitage, con talone pequeño, extraída con percutor blando y pesado.

Figure 4. Core and flakes of débitage. A: rocky basement inside the shelter, with negative of removals (Photo: $A$. Da Costa); $\boldsymbol{B}$ : large and compact débitage flakes, removed by hard direct percussion or soft stone, with thermal contact stigmas, in addition to cortex. They are related to cleaning and preparation of debitage surfaces; C: Initial débitage flakes, longer than wide, with butts also longer but thick, Siret accident and cortex; $\boldsymbol{D}$ : Initial débitage flake, whit small butt, knapped with soft and heavy hammer.

que toda la limpieza inicial se enfocaen la búsqueda de las partes más resistentes, menos alteradas del bloque. Estos productos anuncian la configuración de núcleos específicos (grandes y largos), con una superficie de débitage ideal, estructurada para la extracción de los productos de primera elección las grandes lascas de soporte de instrumentos unifaciales (Rodet et al., 2019).

En lo que se refiere a la segunda cadena operativa de producción de soportes largos, angostos y muy poco espesos, los que serán transformados en instrumentos unifaciales, el análisis se limita solamente a dos instrumentos (en la excavación había 4 con estas características). Por ahora, la regularidad y el patrón de las dos piezas confirman la búsqueda de productos largos y angostos. Se trata de lascas de aproximadamente $10 \mathrm{~cm}$ de $\operatorname{largo}^{5}$, realizadas en una arenisca silicificada, homogénea y de óptima calidad para la talla. Las caras inferiores de estas dos lascas soporte son totalmente planas, sin accidentes tecnológicos. Las caras superiores presentan un negativo largo, en la misma dirección de la lasca soporte, pero podría ser anterior a la extracción de este. Estos elementos indican el uso de una plataforma de percusión preferencial, quizás unipolar y, además, la búsqueda de un espesor muy específico. El negativo dejado por esta extracción anterior recuerda una acanaladura. El resultado final es un soporte largo y muy poco ancho: los espesores en ese sector central de las lascas se aproximan a 0,5-0,3 cm.

El talón de una de las lascas es delgado $(0,3 \mathrm{~cm})$ y, caso estuviera completo, tendría un ancho aproximado de 2 $\mathrm{cm}$ (la segunda lasca no tiene talón, una fisura que parece reciente llevó consigo el sector proximal). Un esquirlado del bulbo llevó una parte del talón, dejando la impresión de que se trata de dos puntos de impacto. El negativo de la esquirla puede ser observado en la cara inferior de la lasca (Figura 5).

El poco espesor de las piezas, junto con el soporte largo y estandarizado, está relacionado a la percusión orgánica. Sería imposible realizar tal extracción con una piedra dura. Sin embargo, los negativos largos que indican el desbaste de lascas en la misma dirección pueden estar relacionados con un epifenómeno: en el momento que el percutor alcanza la plataforma de percusión puede tocar al mismo tiempo en dos puntos (un percutor utilizado, no bien tratado, con superficies heterogéneas, etc.), haciendo salir junto con la lasca soporte, en la parte superior, una lasca con las mismas características buscadas, creando una especie de acanaladura sobre la cara superior del soporte.

\section{Conclusiones}

Los datos observados en las colecciones permiten afirmar la búsqueda de una materia prima muy específica para la producción de estos instrumentos. Tal materia prima responde de manera muy eficaz a la talla, generando productos esperados en el momento de fractura. Estos yacimientos arqueológicos fueron, muy probablemente, frecuentados de manera sistemática y, seguramente, por talladores experimentados. Los tratamientos técnicos específicos de los talones y el gran número de piezas sin accidentes confirman esta hipótesis.

La lectura tecnológica y el programa experimental indican la utilización de la percusión directa con piedra blanda. La presencia de innumerables percutores en arenisca poco silicificada, de dimensiones variadas, complementan esta

\footnotetext{
${ }^{5}$ Dimensiones de los soportes: $10,2 \times 3,1 \times 0,5 \mathrm{~cm}$ y $10,6 \times 3,1 \times 1$ $\mathrm{cm}$.
} 

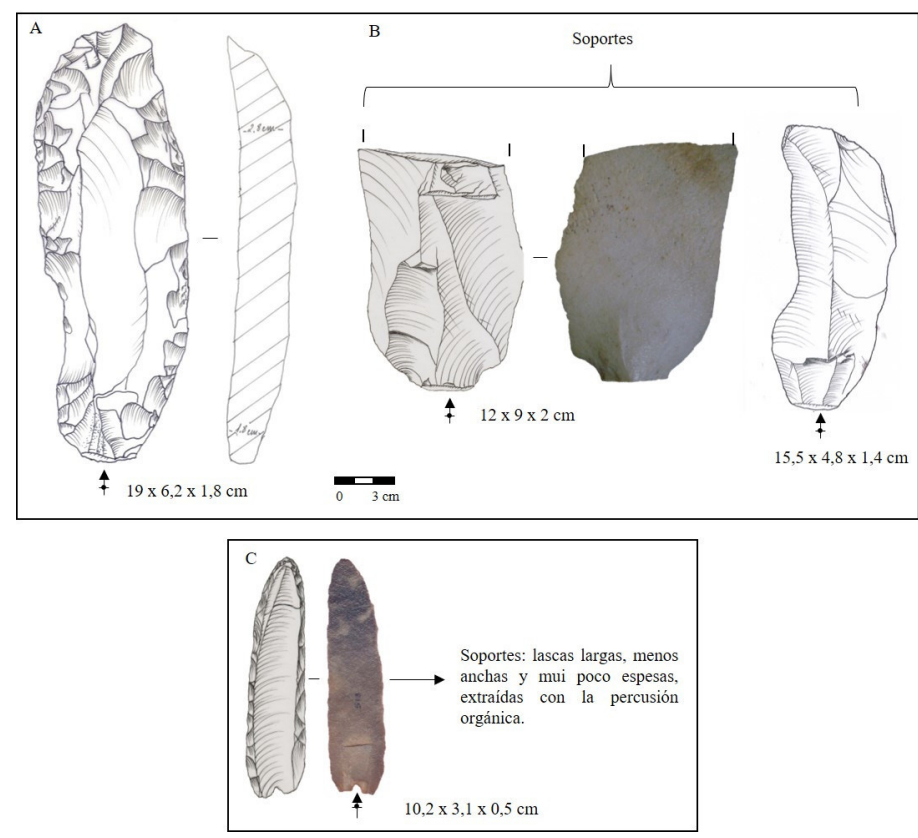

observación. La arenisca poco silicificada constituye un excelente percutor blando, porque absorbe parte de la energía del golpe, evitando así los accidentes.

Las producciones observadas en los niveles antiguos de los yacimientos prehistóricos de la región de Serranópolis son distintas de la homogeneidad prevista para las industrias de la transición del periodo Pleistoceno/Holoceno. En este sentido, se registran conjuntos muy elaborados, con un débitage por percusión blanda y orgánica para producciones de grandes lascas soportes, que serán luego transformados en instrumentos unifaciales de sección plano-convexa o unifaciales muy poco espesos. Las transformaciones serán realizadas por percusión directa de piedra dura y/o por percusión directa de piedra blanda con gestos tangenciales.

Los grandes instrumentos unifaciales de sección planoconvexa conforman una reserva de materia prima y tienen una vida útil muy larga. Son transformados y modificados, pero pensamos que las modificaciones eran previstas desde su concepción inicial. Un instrumento de $20 \mathrm{~cm}$ de largo dispone de al menos $40 \mathrm{~cm}$ de bordes, los cuales pueden ser utilizados para operaciones distintas y los bordes pueden ser reavivados cada vez que sea necesario. Debido a su larga vida útil, estos objetos pueden aparecer en distintos estados técnicos: enteros, al inicio de la utilización; utilizados y reavivados; al final de su vida, menores o fragmentados, transformados o reutilizados. Estos instrumentos están presentes no solamente en Brasil, sino también en Chile, Perú, Venezuela y Colombia (Dillehay, 1999; entre otros) en el mismo momento (Pleistoceno/Holoceno y Holoceno inicial).

La idea de un objeto de sección plano-convexa debe ser muy interesante, porque aparece en Brasil cerca de 12 mil años antes del presente y no desaparece hasta el momento
Figura 5. Las dos principales intenciones productivas observadas en las colecciones: instrumentos unifaciales más y menos espesos. A: Los instrumentos más espesos son sobre grandes lascas alargadas, normalizadas y con un cierto espesor, extraídas con percutores blandos y pesados (orgánicos o de piedra blanda), con gestos específicos, tangenciales. B: Lascas soportes (posibles) para la producción de instrumentos unifaciales; C: Los instrumentos menos espesos son sobre lascas largas, menos anchas y muy poco espesas, extraídas por percusión orgánica.

Figure 5. The two main productive intentions observed in the collections: more and less thick unifacial tools. A: The thicker tools are on large, long, normalized and somewhat thickened flakes, removed with soft and heavy (organic) hammers, or soft stone, with specific gestures, tangencies; B: (Possible) support flake used for the unifacial tools production; $\boldsymbol{C}$ : The less thick tools are on long, less wide and very thin flakes, removed with organic percussion.

del Contacto. Por otro lado, el segundo instrumento presentado en este trabajo (Figura $5 \mathrm{C}$ ) es muy diferente, porque tienne otra concepción, lo cual nos conlleva a concluir que se trata de "familias" de unifaciales.

Finalmente, es importante decir que estas industrias antiguas presentan varios tipos de instrumentos unifaciales: muy espesos, medianamente espesos, poco espesos o muy poco espesos. Siempre aparecen acompañadas por una industria bifacial y también por una industria más simple (lascas o soportes poco transformados). Sumado a ello, se registran instrumentos sobre los restos de débitage, usados sin ninguna transformación.

20 de Diciembre de 2019, Belo Horizonte, Brasil.

\section{Referencias bibliográficas}

Bassi, L. F. y Rodet, M. J. (2011). Abordagens tecnológicas do lascamento de cristal de quartzo. Apresentação e Resumo. Trabalho apresentado no XVI Congresso da Sociedade de Arqueologia Brasileira, Florianópolis. En L. Fernandes y D. Duarte-Talim (Orgs.). Tecnologia lítica na arqueologia brasileira: coletânea de (re) publicações (pp. 218-219). Belo Horizonte: Editor MHNJB-UFMG.

Boëda, E., Clemente-Conte, I., Fontugne, M., Lahaye, C., Pino, M., Felice, G. D., Guidon, N., Hoeltz, S., Lourdeau, A., Pagli, M., Pessis, A.-M., Viana, S., Costa, A. y Douville, E. (2014). A new late Pleistocene archaeological sequence in South America: the Vale da PedraFurada (Piauí, Brazil). Antiquity 88, 927-955.

Bueno, L. (2007). Variabilidade tecnológica dos sítios líticos da região do Lajeado, médio rio Tocantins. Revista do Museu de Arqueologia e Etnologia, Suplemento 4, 215. 
Calderón De La Vara, V. (1969). Nota prévia sobre a arqueologia das regiões central e sudoeste do Estado da Bahia. PRONAPA, Resultados Preliminares do $2^{\circ}$ ano, 1966-1967. Publicações avulsas (10), 135-147.

Chauchat, C. (1991). L'approche technologique dans une étude régionale: le Paijenien de la côte du Pérou. 25 Ansd'études technologiques en préhistoire, 263-273.

Dillehay, T. D. (1999). The late Pleistocene cultures of South America. Evolutionary Anthropology, 7 (6), 206-216.

Hogg, A., G.; Hua, Q.; Blackwell, P. G.; Niu, M.; Buck, C. E.; Guilderson, T. P.; Hwaton, T. J.; Palmer, J. G.; Reimer, P. J.; Reimer, Ron W.; Turney, C. S. M. y Zimmerman, S. R. H. (2013). SHCal13 Southern Hemisphere Calibration, 0-50,000 Years cal BP. Radiocarbon, 55 (4), 1889-1903.

Inizian, M.-L., Reduron-Ballinger, M., Roche, H. y Tixier, J. (2017). Tecnologia da pedra lascada. (Revisión actualizada y ampliada con definiciones y ejemplos brasileros por Rodet, M. J. y Machado, J. R.) Belo Horizonte: Museu de História Natural e Jardim Botânico da UFMG.

Isnardis, A. (2009). Entre as Pedras: as ocupaçõespréhistóricas recentes e os grafismos rupestres de Diamantina, Minas Gerais. (Tesis doctoral). Universidade de São Paulo, São Paulo, Brasil.

Leroi-Gourhan, A. (1964). O gesto e a palavra. Rio de Janeiro: Edições 70, LDA.

Lourdeau, A. (2010). Le techonocomplexo Itaparica: définition tecno-fonctionnelle des industries a piècesfaçonnéesunifacialement à une face plane dans le centre e le nord-est du Brésilpendant la transition Pléistocène-Holocène e Holocèneancien. (Tesis doctoral). Université de Paris Ouest Nanterre La Defense, Paris, Francia.

Martin, G. (2013). Pré-história do nordeste do Brasil. $5^{a}$ ed. Recife: Ed. Universitaria UFPE.

Martins, G. R. y Kashimoto, E. M. (2012). 12.000 anos: arqueologia do povoamento humano no nordeste de Mato Grosso do Sul. Campo Grande: Life Editora.

Mauss, M. (1947). Manuel d'ethnographie. Paris: Petite Bibliothèque Payot.

Moreno de Sousa, J. C. (2014). Cognição e Cultura no mundo material: Os Itaparicas, os Umbus e os "Lagoassantenses". (Tesis de maestría). Universidade de São Paulo, São Paulo, Brasil.

Pelegrin, J. (1991). Les savoir-faire: une très longue histoire. Terrain (16), 107-113.
Pelegrin, J. (2000). Les techniques de débitage laminaire au Tardiglaciaire: critères de diagnose et quelques réflexions. centrale et septentrionale au Tardiglaciaire. Confrontation des modèles régionaux de peuplement. Table-ronde de Nemours, 13-16 mai 1997. Mémoires du Musée de Préhistoire d'lle de France (7), 73-86.

Pelegrin, J. (2005). Les pierres taillées: une historique de leur apport à l'archéologie. En J. D. Wright (Ed.). International Encyclopedia of Social and Behavioral Sciences, vol. 14 (pp.: 8). Oxford: Elsevier.

Prous, A., Fogaça, E. y Alonso, M. (1994). As últimas indústrias líticas do vale do Peruaçu, MG. Revista de Arqueologia, 8 (2), 49-64.

Ramsey, C. B. (2009). Dealing with Outliers and Offsets in Radiocarbon Dating. Radiocarbon, 51 (3), 1023-1045.

Rodet, M. J. (2006). Étude Technologique des industries lithiques taillées du nord de Minas Gerais, Brésil: depuis le passage Pléistocène/Holocène jusqu'au Contact - XVIII'ème siècle. (Tesis doctoral). Université Paris $X$, Nanterre, Paris, Francia.

Rodet, M. J., Duarte-Talim, D. y Bassi, L. F. (2011). Reflexões sobre as primeiras populações do Brasil Central: "Tradição Itaparica". Habitus, 9 (1), 81-100.

Rodet, M. J. Duarte-Talim, D. y Pereira, E. (2017). Algunos aspectos de la industria litica de Monte Alegre, Pará, Brasil. Cuadernos de resumos del IV Encuentro Internacional de Arqueología Amazónica, 22.

Rodet, M. J., Duarte-Talim, D. y Schmtiz, P. I. (2019). As indústrias líticas antigas de Serranópolis (sítios GOJA-03 e GOJA-14). Revista de Arqueologia, 32, 175 - 206.

Roosevelt, A C., Costa, M. L., Machado, C. L, Michab, N., Mercier, N., Valladas, H., Feathers, J., Barnett, W. K., Silveira, M. I., Henderson, J., Sliva, J., Chernoff., B., Reese, D. S., Holman, J. A., Toth, N. y Schick, K. (1996). Paleoindian cave dwellers in the Amazon: the peopling of the Americas. Science, 272, 373-384.

Schmitz P. I.; Rosa, A. O. y Bitencourt, A. L. V. (2004). Arqueologia nos cerrados do Brasil Central - Serranópolis III. Pesquisas (60), pp.287.

Tixier, J. (1978). Méthode pour l'étude des outillages lithiques. Notice sur les travaux scientifiques. (Tesis doctoral). Paris, Université de Paris X, Nanterre, Paris, Francia.

Vieira, D., Ker, J. C., Rodet M. J., Schaefer C. E. G. R. y Teixeira, W. G. (2015). Pedoarqueologia em abrigo quartzitico, sítio Bibocas II, Jequtaí. Ocupação humana no Holoceno tardio, bacia do São Francisco. Teoria \& Sociedade, 23 (1), 167-197. 